\title{
Triglyceride-glucose index is associated with the risk of myocardial infarction: an 11-year prospective study in the Kailuan cohort
}

Xue Tian ${ }^{1,2,3,4+}$, Yingting Zuo ${ }^{1,2,3,4 \dagger}$, Shuohua Chen ${ }^{5}$, Qian Liu ${ }^{6}$, Boni Tao ${ }^{7}$, Shouling Wu ${ }^{5^{*}}$ and Anxin Wang ${ }^{1,2^{*}}$ (])

\begin{abstract}
Background: The triglyceride-glucose (TyG) index, which is a simple surrogate marker of insulin resistance, has been suggested as a contributor of cardiovascular disease. However, evidence on the effect of long-term elevation of the TyG index exposure on myocardial infarction (MI) is limited. The current study aimed to evaluate the association of baseline and long-term elevation of the TyG index exposure with the risk of MI.

Methods: A total of 98,849 participants without MI at baseline (2006) were enrolled from the Kailuan study. The baseline TyG index was calculated as In [fasting triglyceride $(\mathrm{mg} / \mathrm{dL}) \times$ fasting glucose $(\mathrm{mg} / \mathrm{dL}) / 2$ ]. The long-term TyG index was characterized in two ways as follows. The updated mean TyG index was calculated as the mean of TyG index at all previous visits before Ml occurred or the end of follow-up; alternatively, the TyG index was calculated as the number of visits with a high TyG index in 2006, 2008, and 2010, ranging from 0 (no exposure) to 3 (had high TyG index at all three study visits). Hazard ratio (HR) and $95 \%$ confidence interval (Cl) was estimated using multivariable Cox proportion hazard models.
\end{abstract}

Results: During a median follow-up of 11.03 years, 1555 incident Ml occurred. In the multivariable-adjusted model, the risk of Ml increased with quartiles of the baseline and updated mean TyG index, the HR in quartile 4 versus quartile 1 was 2.08 (95\% Cl,1.77-2.45) and 1.58 (1.18-2.12), respectively. Individuals with a high TyG index at all three visits had a 2.04 -fold higher risk $(95 \% \mathrm{Cl}, 1.63-2.56)$ of $\mathrm{Ml}$ compared with no exposure. Subgroup analyses showed that the associations were more pronounced in women than in men $\left(P_{\text {interaction }}=0.0411\right)$.

Conclusions: Elevated levels of the baseline and long-term TyG index are associated with an increased risk of MI. This finding indicates that the TyG index might be useful in identifying people at high risk of developing MI.

Keywords: Triglyceride glucose index, Long-term exposure, Updated mean, Myocardial infarction, Risk factor

*Correspondence: drwus|@163.com; wanganxin@bjtth.org

${ }^{\dagger}$ Xue Tian and Yingting Zuo contribute equally to this work

${ }^{1}$ China National Clinical Research Center for Neurological Diseases,

Fengtai District, Beijing Tiantan Hospital, Capital Medical University, No.119 South 4th Ring West Road, Beijing 100070, China

${ }^{5}$ Department of Cardiology, Kailuan Hospital, North China University of Science and Technology, 57 Xinhua East Rd, Tangshan 063000, China

Full list of author information is available at the end of the article

\section{Background}

Myocardial infarction (MI) is the leading cause of morbidity and mortality of cardiovascular disease (CVD) worldwide, and it accounts for approximately one million deaths in China annually [1,2]. Therefore, prevention of MI through understanding and reduction of risk factors has significant implications for public health and clinical practice.

Insulin resistance (IR) is a critical mechanism of the pathogenesis of diabetes mellitus, and has been extensively demonstrated to be a potential risk factor for CVD

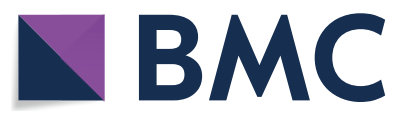

(c) The Author(s) 2021. This article is licensed under a Creative Commons Attribution 4.0 International License, which permits use, sharing, adaptation, distribution and reproduction in any medium or format, as long as you give appropriate credit to the original author(s) and the source, provide a link to the Creative Commons licence, and indicate if changes were made. The images or other third party material in this article are included in the article's Creative Commons licence, unless indicated otherwise in a credit line to the material. If material is not included in the article's Creative Commons licence and your intended use is not permitted by statutory regulation or exceeds the permitted use, you will need to obtain permission directly from the copyright holder. To view a copy of this licence, visit http://creativeco mmons.org/licenses/by/4.0/. The Creative Commons Public Domain Dedication waiver (http://creativecommons.org/publicdomain/ zero/1.0/) applies to the data made available in this article, unless otherwise stated in a credit line to the data. 
because it leads to atherosclerotic aneurysm and small vessel disease. Additionally, IR coexists with hypertension, obesity, and dyslipidemia, and all of these are well-known risk factors of CVD [3-9]. The triglyceride-glucose (TyG) index, which is derived from fasting triglyceride (TG) and fasting blood glucose (FBG) levels, has been proposed as a reliable surrogate marker of IR. Numerous studies have found a positive correlation between the TyG index and cardiovascular risk, including systematic arterial stiffness, carotid atherosclerosis, coronary artery calcification, coronary artery stenosis, symptomatic coronary artery disease, hypertension, and metabolic syndrome [10-16]. Furthermore, growing evidence has indicated that the TyG index is related to morbidity and mortality of CVD in the general population and many patient cohorts, including patients with and those without diabetes [14, 17-22].

Notably, however, the TyG index in most previous studies was measured only using a single time point and the long-term effect of a high TyG index remains unknown. To the best of our knowledge, the TyG index is affected by many biological and environmental factors, a single measurement of a high TyG index does not indicate that the body state has experienced a high TyG index for a long time. Therefore, a single measurement of this index may lead to incorrect classification of risk assessment for MI. Measurements of the TyG index at multiple time points, such as an updated mean or the number of visits with a high TyG index [23, 24], can characterize the longitudinal pattern of TyG index. These multiple measurements can provide a more robust assessment of associations with outcomes than analyses examining relationships using a single measurement of the TyG index, which may be attenuated with extended follow-up.

In the present study, we therefore aimed to investigate the association of an elevated TyG index at baseline and in the long term, where the long term TyG index was estimated as updated mean of TyG index and number of visits with high TyG index, with the risk of MI based on a large community-based prospective cohort study.

\section{Methods}

\section{Study population}

Data were deprived from the Kailuan study, which was a prospective cohort study that was conducted in the Kailuan community in Tanshan City, China. Details of the study design have been described previously [25-27]. In brief, a total of 101,510 participants $(81,110$ men and 20,400 women) aged $18-98$ yeas agreed to participate and completed the first survey from June 2006 to October 2007 and underwent questionnaire assessments, physical examinations, and laboratory tests in the 11 hospitals responsible for health care of the Kailuan community. All participants were then followed up biennially to update the above-mentioned information. In the current study, we used the TyG index at baseline (2006) to predict the subsequent risk of MI. Data analysis was performed from 1 January 2006 to 31 December 2017. We excluded 1317 participants with previous MI and 1344 participants with missing data of TG and FBG levels at baseline. A total of 98,849 participants were enrolled for the analysis of the baseline and updated mean TyG index. In analysis of the number of visits with high a TyG index, we further excluded 42,694 participants without completed data on TG and FBG levels and 703 participants who had MI during 2006 and 2010. Therefore, 55,452 participants were included for the current analysis (Additional file 1: Figure S1).

\section{Data collection and definitions}

Information on demographics, socioeconomic status, medical history, and lifestyle was collected using a selfreport questionnaire by trained staffs, including age, sex, education, income, smoking status, drinking status, physical activity, and a history of disease and medication. Education was classified as illiteracy or primary school, middle school, and high school or above. Income was categorized into $>800$ and $\leq 800$ yuan/month. Smoking and drinking habits were stratified into never, former or current. Physical activity was classified as $\geq 4$ times per week and $\geq 20 \mathrm{~min}$ at a time, $<80 \mathrm{~min}$ per week, or none. Blood pressure was measured in the in the seated position using a mercury sphygmomanometer, and the average of three readings was calculated as systolic blood pressure (SBP) and diastolic blood pressure (DBP).

Fasting blood samples were collected from the antecubital vein after an 8- to 12-h overnight fast. All the plasma samples were assessed using an auto-analyzer (Hitachi 747, Tokyo, Japan) at the central laboratory of Kailuan Hospital. FBG levels were measured using the hexokinase/glucose-6-phosphate dehydrogenase method with the coefficient of variation using blind quality control specimens $<2.0 \%$. Serum total cholesterol (TC), TG, low-density lipoprotein cholesterol (LDL-C), and high-density lipoprotein cholesterol (HDL-C) levels were measured with the enzymatic colorimetric method. High-sensitivity C-reactive protein (hs-CRP) levels were measured with high-sensitivity particle-enhanced immunonephelometry assay. Diabetes was defined as FBG levels $\geq 7.0 \mathrm{mmol} / \mathrm{L}$, any use of glucose-lowing drugs, or any self-reported history of diabetes. Hypertension was defined as $\mathrm{SBP} \geq 140 \mathrm{~mm}$ $\mathrm{Hg}$ or $\mathrm{DBP} \geq 90 \mathrm{~mm} \mathrm{Hg}$, any use of antihypertensive drugs, or a self-reported history of hypertension. Dyslipidemia was defined as any self-reported history or use of 
lipid-lowering drugs, or TC levels $\geq 5.17 \mathrm{mmol} / \mathrm{L}$ or TG levels $\geq 1.69 \mathrm{mmol} / \mathrm{L}$ or LDL-C levels $\geq 3.62 \mathrm{mmol} / \mathrm{L}$ or HDL-C level $\leq 1.04 \mathrm{mmol} / \mathrm{L}$.

\section{Calculation of the baseline and long-term TyG index}

The TyG index was calculated as $\ln$ [(fasting TG $(\mathrm{mg} /$ $\mathrm{dl}) \times$ FBG $(\mathrm{mg} / \mathrm{dl}) / 2$ ] [21]. To determine long-term TyG index patterns of individuals, we calculated the updated mean TyG index and the number of visits with a high TyG index. The updated mean TyG index was calculated using all available TyG index measurements from baseline to the year before MI occurred or to the end of follow-up (2017). An example of this calculation is that incident MI in 2013 was related to the average concentration of TyG index level in 2006, 2008, 2010, and 2012. Those participants who did not develop MI before the end of follow-up (2017) had six times of TyG index measurements and those who developed MI had $\leq$ six TyG index measurements.

In the current study, a high TyG index was defined as a TyG index greater the cutoff value, which was estimated by the receiver operating characteristic curve (ROC). Then the number of visits with a high TyG index was calculated using the TyG index value at visits in 2006, 2008 and 2010. We assigned 1 point to a TyG index higher than the cutoff value, and 0 point for TyG index less than the cutoff value at each visit. Therefore, the number of visits with high a TyG index was ranged from 0 to 3 . An example of this calculation is that 2 points represent a high TyG index twice in the 3 visits.

\section{Follow-up and assessment of MI}

Participants were followed via face-to-face interviews at every 2-year routine medical examination until 31 December 2017 or death. The primary outcome was the first occurrence of MI, either the first nonfatal MI event or death due to MI without a history of MI. The diagnosis of MI events was obtained from biennial personal interviews, the discharge summary from the 11 hospitals, and medical records from medical insurance, using the International Classification of Disease, 10th Revision code I21 for MI. Diagnosis of MI was based on combinations of chest pain symptoms, electrocardiographic signs, and cardiac enzyme levels. The criteria were consistently applied across all 11 hospitals [28].

\section{Statistical analysis}

Continuous variables are described as median and interquartile range (IQR) owing the skewed distribution. Categorical variables are described as frequencies and percentages. The Wilcoxon or Kruskal-Wallis test was used to analyze group differences for continuous variables, and the chi-square test was used for categorical variables. Person-years were determined from the date when the message was collected at baseline to either the date of onset of MI, death, or the date of participating in the last examination in this analysis, whichever came first. The Kaplan-Meier method was performed to evaluate the incidence rate of MI and differences among groups were evaluated by the log-rank test.

Three multivariate Cox proportional hazard regressions were constructed to estimate the association of the baseline and long-term TyG index with the risk of MI by calculating the hazard ratio (HR) and $95 \%$ confidence interval $(\mathrm{CI})$. The validity of the proportionality assumption was verified by scaled Schoenfeld residuals for the baseline TyG index and the number of visits with a high TyG index, and by including a time-dependent covariate with an interaction of the TyG index and a logarithmic function of survival time in the model for the updated mean TyG index. The results of which suggested that the assumptions were not violated (Additional file 1: Table S1). Model 1 was adjusted for age and sex at baseline; Model 2 was further adjusted for education, income, smoking, alcohol abuse, physical activity, and BMI at baseline; Model 3 was further adjusted for SBP, DBP, a history of hypertension, diabetes mellitus, and dyslipidemia, antidiabetic drugs, lipid-lowering drugs, antihypertensive drugs, and HDL-C, LDL-C, and hs-CRP levels at baseline. We added an interaction between the updated mean TyG index and a logarithmic function of survival time in the above-mentioned model for the analysis of the updated mean TyG index and MI. The $P$ values for trend were computed using quartiles or the number of visits with a high TyG index as ordinal variables. We also analyzed the effect of baseline and the updated mean TyG index on MI as a continuous variable using a restricted cubic spline with 5 knots (at the 5th, 25th, 50th, 75th, and 95th percentiles). The optimum cutoff value of the TyG index in case of incident MI was determined using the ROC curve analysis. The best cutoff point for the TyG index was assessed by the maximum value of the Youden index, which was calculated as sensitivity + specificity -1 .

Additional analyses were performed to evaluate the robustness of the association of the TyG index with the risk of MI. First, stratified analyses according to baseline age ( $<60$ and $\geq 60$ years), sex, diabetes (no and yes), and BMI $\left(<28\right.$ and $\left.\geq 28 \mathrm{~kg} / \mathrm{m}^{2}\right)$ were used to examine the consistence of the effect of a high TyG index (quartile 4 group) for the risk of MI. Second, to evaluate the robustness of our main results, sensitivity analysis was conducted by excluding all deaths during the followup period $(n=9640)$. Another sensitivity analysis was performed by excluding participants with abnormal 
FBG levels $(\geq 7.0 \mathrm{mmol} / \mathrm{L})$ or abnormal TG levels $(\geq 1.7 \mathrm{mmol} / \mathrm{L})$ at baseline $(\mathrm{n}=35346)$.

All analyses were conducted using SAS version 9.4 (SAS Institute Inc., Cary, NC, USA). A two-sided $P<0.05$ was considered statistically significant.

\section{Results}

Baseline characteristics

A total of 98849 participants were included in the current study. The median age was 51.81 years (IQR, $43.60-59.23)$ and 78825 (79.75\%) were men. Baseline characteristics by quartiles of the TyG index are shown in Table 1. Participants with a higher TyG index tended to be older, men, less educated, have higher income, more current smokers and alcohol drinkers, a higher prevalence of diabetes, hypertension, and dyslipidemia, take more antihypertensive, antidiabetic, and lipid-lowering drugs, have higher SBP, DBP, FBG, TC, TG, LDL-C, HDL-C, and hs-CRP levels compared with participants in quartile 1 group. Similar results were observed when participants were categorized by the updated mean TyG index (Additional file 1: Table S2).

Table 1 Baseline characteristics of participants according to quartiles of TyG index

\begin{tabular}{|c|c|c|c|c|c|c|}
\hline \multirow[t]{2}{*}{ Characteristics } & \multirow[t]{2}{*}{ Overall } & \multicolumn{4}{|c|}{ Quartiles of TyG index } & \multirow[t]{2}{*}{$P$ value } \\
\hline & & Q1 & Q2 & Q3 & Q4 & \\
\hline No. of participants & 98,849 & 24,712 & 24,716 & 24,708 & 24,713 & \\
\hline TyG index & $8.58(8.18-9.06)$ & $7.91(7.70-8.06)$ & $8.39(8.29-8.48)$ & $8.79(8.68-8.91)$ & $9.46(9.23-9.82)$ & $<0.0001$ \\
\hline Age, years & $51.81(43.60-59.23)$ & $50.77(41.81-58.91)$ & $51.82(43.60-59.18)$ & $52.36(44.22-59.70)$ & $52.16(44.44-58.96)$ & $<0.0001$ \\
\hline Men, n (\%) & 78,835 (79.75) & $18,283(73.98)$ & $19,634(79.44)$ & $20,104(81.37)$ & $20,814(84.22)$ & $<0.0001$ \\
\hline $\begin{array}{l}\text { High school or above, } \\
\text { n (\%) }\end{array}$ & 19,189 (20.09) & $5781(24.32)$ & $4586(19.12)$ & $4561(19.12)$ & $4261(17.84)$ & $<0.0001$ \\
\hline $\begin{array}{c}\text { Income }>800 \text { RMB/ } \\
\text { month, } \mathrm{n}(\%)\end{array}$ & $13,659(14.31)$ & $3699(15.57)$ & $3223(13.45)$ & $3357(14.08)$ & $3380(14.16)$ & $<0.0001$ \\
\hline $\begin{array}{l}\text { Body mass index, } \\
\mathrm{kg} / \mathrm{m}^{2}\end{array}$ & $24.84(22.60-27.22)$ & $23.14(21.11-25.34)$ & $24.44(22.39-26.64)$ & $25.43(23.4-27.67)$ & $26.30(24.22-28.41)$ & $<0.0001$ \\
\hline $\begin{array}{l}\text { Systolic blood pres- } \\
\text { sure, } \mathrm{mm} \mathrm{Hg}\end{array}$ & $\begin{array}{l}130.00(119.30- \\
141.30)\end{array}$ & $\begin{array}{l}120.00(110.00- \\
136.00)\end{array}$ & $\begin{array}{l}129.30(117.30- \\
140.00)\end{array}$ & $\begin{array}{l}130.00(120.00- \\
145.00)\end{array}$ & $\begin{array}{l}132.00(120.00- \\
150.00)\end{array}$ & $<0.0001$ \\
\hline $\begin{array}{l}\text { Diastolic blood pres- } \\
\text { sure, } \mathrm{mm} \mathrm{Hg}\end{array}$ & 80.00 (78.70-90.00) & $80.00(70.70-85.00)$ & $80.00(77.30-90.00)$ & $81.00(79.30-90.00)$ & 85.00 (80.00-92.00) & $<0.0001$ \\
\hline Current smoker, n (\%) & $33,064(34.34)$ & $8014(33.50)$ & $7750(32.10)$ & $8284(34.39)$ & $9016(37.37)$ & $<0.0001$ \\
\hline $\begin{array}{l}\text { Current alcohol use, } \\
\text { n (\%) }\end{array}$ & $35,945(37.32)$ & $8941(37.36)$ & $8415(34.85)$ & $8921(37.02)$ & $9668(40.07)$ & $<0.0001$ \\
\hline $\begin{array}{l}\text { Active physical activ- } \\
\text { ity, n (\%) }\end{array}$ & 87,005 (91.28) & 21,660 (91.33) & $21,964(91.77)$ & $21,641(90.85)$ & $21,740(91.19)$ & 0.0046 \\
\hline $\begin{array}{l}\text { Diabetes Mellitus, } \\
\text { n (\%) }\end{array}$ & $3033(3.07)$ & $182(0.74)$ & $348(1.41)$ & $667(2.70)$ & $1836(7.43)$ & $<0.0001$ \\
\hline Hypertension, n (\%) & $12,300(12.44)$ & $1899(7.685)$ & $2549(10.31)$ & $3479(14.08)$ & $4373(17.70)$ & $<0.0001$ \\
\hline Dyslipidemia, n (\%) & $5757(5.82)$ & $751(3.04)$ & $1044(4.22)$ & $1613(6.53)$ & $2349(9.50)$ & $<0.0001$ \\
\hline $\begin{array}{l}\text { Antihypertensive } \\
\text { drugs, n (\%) }\end{array}$ & $2311(2.34)$ & $137(0.55)$ & $246(1.00)$ & $513(2.08)$ & $1415(5.73)$ & $<0.0001$ \\
\hline $\begin{array}{l}\text { Antidiabetic drugs, } \\
\text { n (\%) }\end{array}$ & $845(0.85)$ & $97(0.39)$ & $156(0.63)$ & $201(0.81)$ & $391(1.58)$ & $<0.0001$ \\
\hline $\begin{array}{l}\text { Lipid-lowering drugs, } \\
\text { n (\%) }\end{array}$ & 10,670 (10.79) & 1647 (6.66) & $2188(8.85)$ & $3050(12.34)$ & $3785(15.32)$ & $<0.0001$ \\
\hline $\begin{array}{l}\text { Fasting plasma glu- } \\
\text { cose, mmol/L }\end{array}$ & $5.11(4.66-5.71)$ & $4.78(4.37-5.20)$ & $5.01(4.62-5.46)$ & $5.24(4.80-5.82)$ & $5.67(5.02-6.90)$ & $<0.0001$ \\
\hline $\begin{array}{l}\text { Total cholesterol, } \\
\text { mmol/L }\end{array}$ & $4.92(4.28-5.59)$ & $4.59(4.03-5.20)$ & $4.89(4.29-5.46)$ & $5.07(4.47-5.71)$ & $5.19(4.44-5.94)$ & $<0.0001$ \\
\hline Triglycerides, mmol/L & $1.27(0.89-1.93)$ & $0.70(0.58-0.82)$ & $1.10(0.98-1.22)$ & $1.56(1.36-1.79)$ & $2.76(2.18-3.90)$ & $<0.0001$ \\
\hline $\mathrm{HDL}-\mathrm{C}, \mathrm{mmol} / \mathrm{L}$ & $1.50(1.28-1.76)$ & $1.54(1.30-1.80)$ & $1.52(1.31-1.77)$ & $1.48(1.27-1.74)$ & $1.47(1.25-1.74)$ & $<0.0001$ \\
\hline LDL-C, mmol/L & $2.33(1.82-2.83)$ & $2.13(1.60-2.72)$ & $2.37(1.91-2.82)$ & $2.41(1.97-2.90)$ & $2.38(1.82-2.88)$ & $<0.0001$ \\
\hline $\mathrm{Hs}-\mathrm{CRP}, \mathrm{mg} / \mathrm{dL}$ & $0.80(0.30-2.20)$ & $0.60(0.21-1.90)$ & $0.73(0.30-2.00)$ & $0.90(0.34-2.20)$ & $1.04(0.40-2.66)$ & $<0.0001$ \\
\hline
\end{tabular}




\section{Association of the baseline and updated mean TyG index with the risk of $\mathrm{MI}$}

During a median follow-up of 11.03 years (IQR, 10.7111.21), 1555 (1.57\%) participants developed MI. The association between the TyG index and the risk of MI is shown in Table 2. The incidence of MI substantially increased with increasing TyG index quartiles, from 0.84 (95\% CI, 0.74-0.96) in quartile 1 to 2.28 (95\% CI, 2.112.48) per 1000 person-years in quartile 4. Kaplan-Meier curves also showed that participants in quartile 4 of the baseline and updated mean TyG index had a higher risk for MI events than participants in the other groups during the follow-up period (log-rank test, $P<0.0001$; Fig. 1a and $b$ ).

The risk of MI increased over time by baseline TyG quartiles and remained significant even after adjustment for potential confounding factors, the fully adjusted HRs (model 3) were 1.26 (95\% CI, 1.06-1.50), 1.60 (1.36-1.89), and 2.08 (1.77-2.45) for quartiles 2,3 and 4, respectively, versus quartile 1 of the baseline TyG index

Table $2 \mathrm{HR}(95 \% \mathrm{Cl})$ for risk of myocardial infarction according to quartiles of the TyG index

\begin{tabular}{|c|c|c|c|c|c|c|}
\hline \multirow[t]{2}{*}{ Variables } & \multicolumn{4}{|c|}{ Quartiles of the TyG index } & \multirow[t]{2}{*}{ Per 1 unit increase } & \multirow[t]{2}{*}{$P$ for trend } \\
\hline & Q1 & Q2 & Q3 & Q4 & & \\
\hline \multicolumn{7}{|l|}{ Baseline TyG index } \\
\hline Case, n (\%) & $220(0.89)$ & $315(1.27)$ & $430(1.74)$ & $590(2.39)$ & & \\
\hline Incidence rate, per 1000 person-y & $0.84(0.74-0.96)$ & $1.21(1.08-1.35)$ & $1.65(1.51-1.82)$ & $2.28(2.11-2.48)$ & & \\
\hline Model 1 & Reference & $1.39(1.17-1.65)$ & $1.88(1.60-2.21)$ & $2.69(2.30-3.14)$ & $1.61(1.51-1.72)$ & $<0.0001$ \\
\hline Model 2 & Reference & $1.33(1.12-1.58)$ & $1.73(1.47-2.05)$ & $2.41(2.06-2.83)$ & $1.54(1.43-1.65)$ & $<0.0001$ \\
\hline Model 3 & Reference & $1.26(1.06-1.50)$ & $1.60(1.36-1.89)$ & $2.08(1.77-2.45)$ & $1.43(1.33-1.53)$ & $<0.0001$ \\
\hline Sensitivity analysis ${ }^{a}$ & Reference & $1.24(1.01-1.51)$ & $1.62(1.34-1.96)$ & $2.02(1.67-2.44)$ & $1.39(1.28-1.52)$ & $<0.0001$ \\
\hline Sensitivity analysis ${ }^{b}$ & Reference & $1.22(1.02-1.46)$ & $1.50(1.24-1.82)$ & $3.60(1.48-8.80)$ & $1.63(1.32-2.02)$ & $<0.0001$ \\
\hline \multicolumn{7}{|l|}{ Updated mean TyG index } \\
\hline Case, n (\%) & $264(1.07)$ & $318(1.29)$ & $421(1.70)$ & $552(2.23)$ & & \\
\hline Incidence rate, per 1000 person-y & $1.02(0.90-1.15)$ & $1.22(1.09-1.36)$ & $1.61(1.46-1.36)$ & $2.13(1.96-2.32)$ & & \\
\hline Model 1 & Reference & $1.20(1.00-1.43)$ & $1.54(1.24-1.94)$ & $2.10(1.57-2.79)$ & $1.74(1.48-2.04)$ & $<0.0001$ \\
\hline Model 2 & Reference & $1.13(0.95-1.36)$ & $1.42(1.14-1.78)$ & $1.86(1.39-2.49)$ & $1.64(1.39-1.93)$ & $<0.0001$ \\
\hline Model 3 & Reference & $1.07(0.90-1.29)$ & $1.30(1.03-1.62)$ & $1.58(1.18-2.12)$ & $1.49(1.26-1.76)$ & $<0.0001$ \\
\hline Sensitivity analysis ${ }^{a}$ & Reference & $1.01(0.78-1.20)$ & $1.24(1.02-1.58)$ & $1.42(1.13-2.05)$ & $1.47(1.32-1.63)$ & $<0.0001$ \\
\hline Sensitivity analysis ${ }^{b}$ & Reference & $1.17(0.86-1.59)$ & $1.21(0.90-1.64)$ & $1.80(1.36-2.38)$ & $1.86(1.25-2.57)$ & $<0.0001$ \\
\hline
\end{tabular}

$\mathrm{Cl}$ confidence interval, $H R$ hazard ratio, TyG triglyceride glucose

Model 1, adjusted for age and sex at baseline

Model 2, adjusted for variables in model 1 plus level of education, income, smoking, alcohol abuse, physical activity, and BMI at baseline

Model 3, adjusted for variables in model 2 plus SBP, DBP, a history of hypertension, diabetes mellitus, and dyslipidemia, antidiabetic drugs, lipid-lowering drugs, antihypertensive drugs, HDL-C, LDL-C, and hs-CRP at baseline

a Sensitivity analysis was adjusted for variables in model 3 and further excluded all deaths during the follow-up visits

b Sensitivity analysis was excluded those with abnormal FBG ( $\geq 7.0 \mathrm{mmol} / \mathrm{L})$ or abnormal TG level ( $\geq 1.7 \mathrm{mmol} / \mathrm{L})$ at baseline, and adjusted for covariates in Model 3

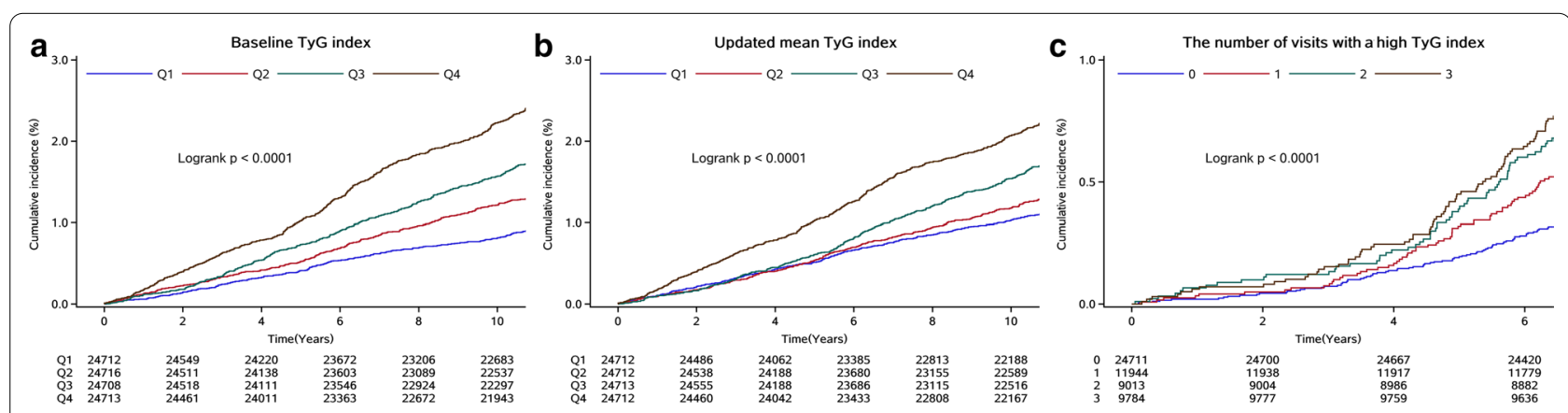

Fig. 1 Kaplan-Meier estimation of myocardial infarction by baseline and updated mean of TyG index. TyG triglyceride-glucose 
( $P$ for trend $<0.0001)$. The association remained significant in analyses using the updated mean TyG index, the adjusted HR in quartile 4 versus quartile 1 was 1.58 (95\% CI, 1.18-2.12, $P$ for trend $<0.0001$; Table 2). Sensitivity analysis yielded the similar results when all the deaths during the follow-up period were excluded, and when the analysis was restricted to participants with normal FBG and TG levels.

When the TyG index was treated as a continuous variable, a per 1 unit increase of the baseline TyG index was associated with a $43 \%$ higher risk of MI (HR, 1.43; 95\% CI, 1.33-1.53). Multivariable adjusted spline regression models showed a J-shaped association between the baseline TyG index and the risk of MI (Fig. 2a). All the significant results persisted in the analyses using the updated mean TyG index (Table 2, Fig. 2b).

\section{Association between the number of visits with a high TyG index and the risk of $\mathrm{MI}$}

In this analysis, we compared baseline characteristics of participants who were included and those who were excluded owing to missing data (Additional file 1: Table S3). There were significant clinical differences regarding baseline characteristics of age, sex, education, SBP, DBP, drinking status, and LDL-C levels between the two groups.

Among the 55,452 enrolled participants, the area under the curve of the TyG index for MI was 0.59 (95\% CI $0.57-0.60$ ). The cutoff value we determined for the TyG index for incident MI was 8.83 (Additional file 1: Figure S2). Participants with all three study visits with a high TyG index had a higher risk of MI than the other groups (log-rank test, $P<0.0001$, Fig. 1c). Significant associations remained even after adjustment for potential confounders, the HRs in the fully adjusted model were 1.54 (95\% CI, 1.23-1.93), 1.78 (1.41-2.26), and 2.04 (1.63-2.56) for participants with a high TyG index once, twice, three times of the three visits, respectively, compared to those with a TyG index less than the cutoff value at all the three visits. Sensitivity analysis yielded similar results (Table 3).

\section{Subgroup analysis}

Results of subgroup analyses are shown in Table 4. Generally, a high TyG index (quartile 4) was significantly associated with the risk of MI across various subgroups. There was a significant interaction in the sex subgroup ( $P$ for interaction $=0.0411$ in model 3 ). The hazards of a high TyG index on MI were more prominent in women (HR, 3.77; 95\% CI, 1.86-7.64) than men (HR, 1.93; 95\% CI, 1.63-2.29).

\section{Discussion}

In this large community-based cohort study, we observed that a high baseline and long-term TyG index were associated with the risk of MI, even in those with normal FBG and TG levels. Specifically, participants in the quartile 4 group of the baseline and updated mean TyG index, and those had three times of a high TyG index had a higher risk of MI than the other groups. Additionally, subgroup analyses showed there was a significant interaction between sex and the TyG index, the effect of a high TyG index on the risk of MI was more pronounced in women compared with men. The results obtained from this large, prospective, observational study have important implications for prevention of MI.
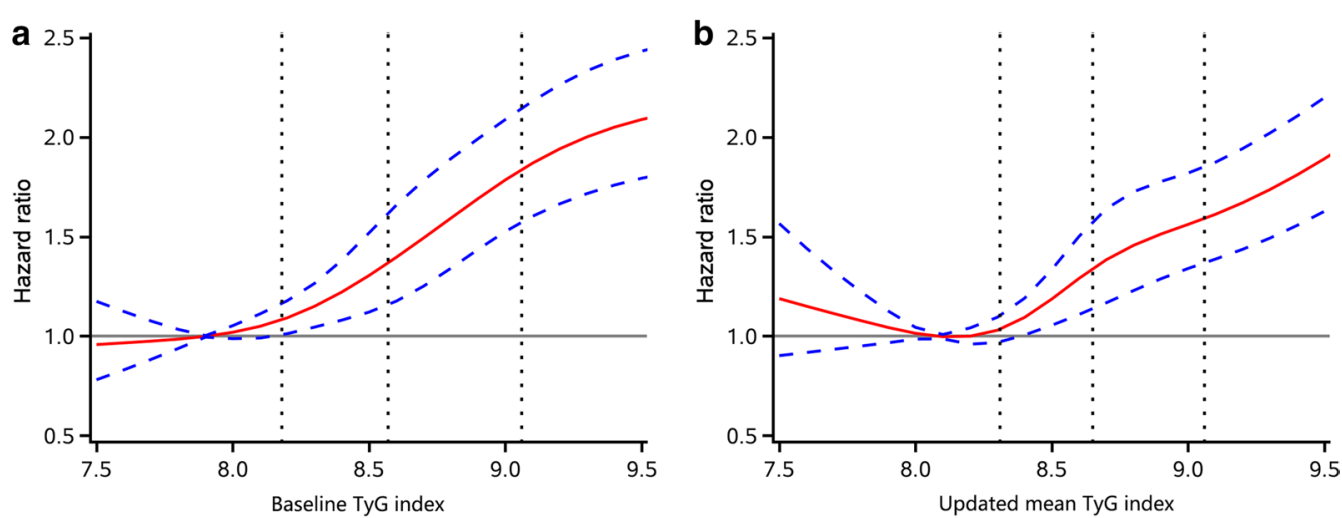

Fig. 2 Multivariable-adjusted hazard ratios for Ml based on restricted cubic spines with 5 knots at 5th, 25th, 50th, 75th, and 95th percentiles of a baseline TyG index and $\mathbf{b}$ updated mean TyG index. Abbreviation: TyG, triglyceride-glucose. Red line represent references for hazard ratios, and blue lines represent 95\% confidence interval. Adjusted for age, sex, education, income, smoking, alcohol abuse, physical activity, and body mass index, systolic blood pressure, diastolic blood pressure, a history of hypertension, diabetes mellitus, and dyslipidemia, antidiabetic drugs, lipid-lowering drugs, antihypertensive drugs, HDL cholesterol, LDL cholesterol, and hs-CRP 
Table 3 HR (95\% CI) for risk of myocardial infarction according to number of visits with a high TyG index

\begin{tabular}{|c|c|c|c|c|c|}
\hline \multirow[t]{2}{*}{ Variables } & \multicolumn{5}{|c|}{ Number of visits with a high TyG index* } \\
\hline & 0 & 1 & 2 & 3 & $P$ for trend \\
\hline Case, n (\%) & $173(0.70)$ & $139(1.40)$ & $126(1.40)$ & $164(1.68)$ & \\
\hline $\begin{array}{l}\text { Incidence rate, per } 1000 \\
\text { person-y }\end{array}$ & $0.65(0.56-0.75)$ & $1.07(0.91-1.27)$ & $1.29(1.09-1.54)$ & $1.55(1.33-1.81)$ & \\
\hline Model 1 & Reference & $1.69(1.36-2.12)$ & $2.10(1.67-2.64)$ & $2.52(2.03-3.12)$ & $<0.0001$ \\
\hline Model 2 & Reference & $1.61(1.28-2.01)$ & $1.90(1.50-2.40)$ & $2.21(1.77-2.76)$ & $<0.0001$ \\
\hline Model 3 & Reference & $1.54(1.23-1.93)$ & $1.78(1.41-2.26)$ & $2.04(1.63-2.56)$ & $<0.0001$ \\
\hline Sensitivity analysis & Reference & $1.48(1.16-1.91)$ & $1.78(1.37-2.31)$ & $2.16(1.69-2.78)$ & $<0.0001$ \\
\hline
\end{tabular}

Cl confidence interval, $H R$ hazard ratio, TyG triglyceride glucose

* High TyG index was defined as TyG index $\geq 8.83$

Model 1, adjusted for age and sex at baseline

Model 2, adjusted for variables in model 1 plus level of education, income, smoking, alcohol abuse, physical activity, and BMI at baseline

Model 3, adjusted for variables in model 2 plus SBP, DBP, a history of hypertension, diabetes mellitus, and dyslipidemia, antidiabetic drugs, lipid-lowering drugs, antihypertensive drugs, $\mathrm{HDL}-\mathrm{C}, \mathrm{LDL}-\mathrm{C}$, and hs-CRP at baseline

Sensitivity analysis was adjusted for variables in model 3 and further excluded all deaths during the follow-up visits

Table 4 Subgroup analysis for the risk of myocardial infarction by baseline TyG index (impact of TyG Q4)

\begin{tabular}{|c|c|c|c|c|c|c|c|}
\hline \multirow[t]{2}{*}{ Variables } & \multirow[t]{2}{*}{ Group } & \multicolumn{2}{|l|}{ Model 1} & \multicolumn{2}{|l|}{ Model 2} & \multicolumn{2}{|l|}{ Model 3} \\
\hline & & $\mathrm{HR}(95 \% \mathrm{Cl})$ & $P_{\text {interaction }}$ & $\mathrm{HR}(95 \% \mathrm{Cl})$ & $P_{\text {interaction }}$ & $\mathrm{HR}(95 \% \mathrm{Cl})$ & $P_{\text {interaction }}$ \\
\hline \multicolumn{8}{|l|}{ Age } \\
\hline$<60$ years & Q4 & $2.69(2.15-3.35)$ & 0.1428 & $2.39(1.90-3.00)$ & 0.2935 & $2.12(1.69-2.68)$ & 0.3000 \\
\hline$\geq 60$ years & Q4 & $2.43(1.95-3.02)$ & & $2.25(1.79-2.83)$ & & $1.94(1.53-2.45)$ & \\
\hline \multicolumn{8}{|l|}{ Sex } \\
\hline Women & Q4 & $5.29(2.70-10.37)$ & 0.0128 & $5.13(2.59-10.18)$ & 0.0164 & $3.77(1.86-7.64)$ & 0.0411 \\
\hline Men & Q4 & $2.47(2.11-2.90)$ & & $2.20(1.86-2.60)$ & & $1.93(1.63-2.29)$ & \\
\hline \multicolumn{8}{|l|}{ Diabetes } \\
\hline No & Q4 & $2.57(2.19-3.02)$ & 0.1352 & $2.28(1.93-2.69)$ & 0.1430 & $2.09(1.77-2.47)$ & 0.1138 \\
\hline Yes & Q4 & $1.56(0.68-3.57)$ & & $1.64(0.71-3.80)$ & & $1.54(0.66-3.57)$ & \\
\hline \multicolumn{8}{|l|}{$B M I$} \\
\hline$<28 \mathrm{~kg} / \mathrm{m}^{2}$ & Q4 & $2.65(2.23-3.14)$ & 0.3394 & $2.43(2.04-2.90)$ & 0.2573 & $2.08(1.74-2.49)$ & 0.4420 \\
\hline$\geq 28 \mathrm{~kg} / \mathrm{m}^{2}$ & Q4 & $2.30(1.48-3.58)$ & & $2.30(1.48-3.58)$ & & $2.07(1.32-3.23)$ & \\
\hline
\end{tabular}

$B M I$ body mass index, $\mathrm{Cl}$ confidence interval, $H R$ hazard ratio, Ty $G$ triglyceride glucose

Model 1, adjusted for age and sex at baseline other than the variable for stratification

Model 2, adjusted for variables in model 1 plus level of education, income, smoking, alcohol abuse, physical activity, and BMI at baseline other than the variable for stratification

Model 3, adjusted for variables in model 2 plus SBP, DBP, a history of hypertension, diabetes mellitus, and dyslipidemia, antidiabetic drugs, lipid-lowering drugs, antihypertensive drugs, HDL-C, LDL-C, and hs-CRP at baseline other than the variable for stratification

IR is defined as a decrease in the efficiency of insulin in promoting glucose uptake and utilization and has been considered as an important risk factor for CVD [3-9]. Evaluations of IR requires sophisticated methods, which are not available for use in daily clinical practice [11]. Therefore, a number of surrogate markers of IR have been proposed and compared with the gold standard of the hyperinsulinemic-euglycemic clamp [29]. Hemeostasis model assessment of IR (HOMA-IR), which is calculated by fating insulin and glucose, is commonly used for testing IR. However, the insulin concentrations are not routinely measured in clinical practice, leading to an HOMA-IR that is inappropriate for clinical practice on a large scale. Therefore, researchers began to study the TyG index, which is a simple, cost-effective, reproducible, reliable, and valid surrogate marker of IR. Additionally, the TyG index is well related to the hyperinsulinemiceuglycemic clamp and HOMA-IR [30, 31]. 
Our finding that a high baseline TyG index was associated with an increased risk of developing $\mathrm{MI}$ is supported by several cross-sectional, case-control, prospective ad retrospective studies in general and patients cohorts [14, 17-22]. The National Health and Nutrition Examination Survey III study of 6093 participants showed that a high TyG index had a $21 \%$ higher risk of subclinical myocardial injury [20]. The Vascular Metabolic CUN cohort study of 5014 patients suggested that the TyG index can be used to identify high-risk cardiovascular events at an early stage with a HR of 2.32 (95\% CI: 1.65-3.26) for the highest quintile [17]. Another retrospective cohort study including 6078 subjects aged over 60 years demonstrated that an the risk of developing CVD was 1.72-fold higher in the quartile 4 group of TyG index, compared with quartile 1 [18]. Two Chinese cohort studies reported that the TyG index was associated with adverse cardiovascular outcomes in patients with type 2 diabetes mellitus or with acute coronary syndrome who underwent percutaneous coronary intervention [21, 22]. In line with previous studies, our study of 98,849 participants with sufficient statistical power showed that individuals in the quartile 4 group of the baseline TyG index had a 2.08-fold higher risk of MI compared with the quartile 1 group. After adjusted all potential covariates, this significant association of a higher risk of $\mathrm{MI}$ in the quartile 4 group persisted in those with normal FBG and TG levels.

Most previous studies relied on TyG index measurements from a single time point, which is an important limitation because of variability of the TyG index level over time. Therefore, measurements of long-term exposure of TyG provide more reliable and robust results. In the current study, the long-term TyG index was estimated at multiple time points with the updated mean TyG index and the number of visits with a high TyG index. Recent studies have used these methods of other parameters to predict CVD as follows. The Clinical Practice Research Datalink study showed that time-updated hemoglobin $A_{1 c}$ values showed a stronger relation with $M I$ than baseline hemoglobin $A_{1 c}$ values [32]. Another Kailuan study revealed that participants with three visits with high hs-CRP levels had a 38\% higher risk of CVD and 1.13 higher risk of MI than those without high hs-CRP levels [33]. Using the methods mentioned above, our study showed that the risk of MI increased with quartiles of the updated mean TyG index and the number of visits with a high TyG index. This finding indicated that a high TyG index in the long term was also associated with the risk of MI. Taken together, our findings suggested that not only a high baseline TyG index, but also a high TyG index in the long term, can predict a high risk of MI. Therefore, monitoring the TyG index over time in clinical practice is important.
Another important finding of our study is that the association between the TyG index and the risk of MI was more pronounced in women than men, which is in accordance with previous studies [34, 35]. A cohort study showed that higher fasting serum insulin levels and HOMA-IR were associated with incident hypertension in women, but not in men [34]. Moreover, the Coronary Artery Calcification in Type 1 Diabetes cohort study found that type 1 diabetes affected adipose and skeletal muscle insulin sensitivity to a greater extent in women than in men, which might have contributed to the greater relative increase in cardiovascular risk in women [35]. A possible mechanism for this finding may be a role of estrogen in premenopausal cardiovascular protection and enhanced insulin sensitivity. Following menopause, women's cardiovascular protection decreases and IR increases [36].

Although the potential mechanism underlying the association of the TyG index with the risk of MI has not been elucidated, there are several speculations summarized as follows. First, studies have shown that FBG levels mainly reflects IR from the liver, whereas fasting TG levels mainly reflect IR from adipose cells. Therefore, the TyG index may reflect IR from two aspects. IR is significantly associated with endothelial dysfunction, oxidative stress, cardiovascular remodeling, coagulation imbalance, and the inflammation response [4, 20,37]. Second, the TyG index is linked to IR, which can induce an imbalance in glucose metabolism that generates chronic hyperglycemia, and can also alter lipid metabolism and lead to dyslipidemia. These metabolic changes may contribute to the development of MI [4]. Third, the TyG index is related to arterial stiffness as evaluated by pulse pressure, brachial-ankle pulse wave velocity, and carotid-femoral pulse wave velocity, all of which are major risk factors of MI $[10,11]$.

\section{Strengths and limitations}

The strengths of the study include its prospective design, large community-based sample, long follow-up period, and the availability of repeated measurements of the TyG index. However, the current study also has several limitations. First, owing to the observational nature of the study, we could not establish a causal association between the TyG index and the risk of MI. Therefore, our findings need to be confirmed in future studies. Furthermore, although potential cardiac risk factors were adjusted for, we still cannot exclude the possibility of residual or unmeasured confounding given the observational study design of the present analysis. Second, further studies are required to determine whether the findings from the present study including only Chinese patients can be extrapolated to other ethnic groups. Third, due to the shortage 
of records on insulin, we could not compare the TyG index with HOMA-IR and the hyperinsulinemic-euglycemic clamp test.

\section{Conclusions}

In conclusion, our study shows that an elevated TyG index at baseline and in the long-term is independently associated with increased risk of MI, especially in women. Our findings indicate that this simple index may be useful for identifying individuals at high risk of developing MI at an early stage, and emphasize the importance of monitoring the TyG index in the long term in clinical practice.

\section{Supplementary Information}

The online version contains supplementary material available at https://doi. org/10.1186/s12933-020-01210-5.

Additional file 1: Table S1. Baseline characteristics of excluded and included participants. Table S2. Proportionality assumptions test. Table S3. Baseline characteristics according to quartiles of updated mean TyG index. Figure S1. Flowchart of the study. Figure S2. Receiver operative characteristics curve and cutoff value of triglyceride-glucose index for incident myocardial infarction.

\section{Abbreviations}

BMI: Body mass index; Cl: Confidence interval; CVD: Cardiovascular disease: DBP: Diastolic blood pressure; FBG: Fasting blood glucose; HDL-C: High-density lipoprotein cholesterol; HOMA-IR: Hemeostasis model assessment of IR; HR: Hazard ratio; hs-CRP: High-sensitivity C-reactive protein; IQR: Interquartile range; IR: Insulin resistance; LDL-C: Low-density lipoprotein cholesterol; MI: Myocardial infarction; ROC: Receiver operative characteristic; SBP: Systolic blood pressure; TC: Total cholesterol; TG: Triglyceride; TyG: Triglyceride-glucose.

\section{Acknowledgements}

We thank all study participants, their relatives, the members of the survey teams at the 11 regional hospitals of the Kailuan Medical Group; and the project development and management teams at the Beijing Tiantan Hospital and the Kailuan Group.

\section{Authors' contributions}

$X T$ and $Y Z$ made substantial contributions to study design, data collection, data analysis and manuscript writing. SW and AW made substantial contributions to study design and intellectual direction. SC, QL, and BT made contributions to data collection. All authors read and approved the final manuscript.

\section{Funding}

This work was supported by National Key R\&D Program of China (2017YFC1310902), Young Elite Scientists Sponsorship Program by CAST (2018QNRC001), Beijing Municipal Administration of Hospitals Incubating Program (PX2020021), and Beijing Excellent Talents Training Program (2018000021469G234)

\section{Availability of data and materials}

The datasets used and/or analyzed during the current study are available from the corresponding author on reasonable request.

\section{Ethics approval and consent to participate}

The study was performed according to the guidelines of the Helsinki Declaration and was approved by the Ethics Committee of Kailuan General Hospital (Approval Number: 2006-05) and Beijing Tiantan Hospital (Approval Number: 2010-014-01). All participants were agreed to take part in the study and provided informed written consent.
Consent for publication

Not applicable.

\section{Competing interests}

These authors declare that they have no conflicts of interests.

\section{Author details}

${ }^{1}$ China National Clinical Research Center for Neurological Diseases, Fengtai District, Beijing Tiantan Hospital, Capital Medical University, No.119 South 4th Ring West Road, Beijing 100070, China. ${ }^{2}$ Department of Neurology, Beijing Tiantan Hospital, Capital Medical University, Beijing, China. ${ }^{3}$ Department of Epidemiology and Health Statistics, School of Public Health, Capital Medical University, Beijing, China. ${ }^{4}$ Beijing Municipal Key Laboratory of Clinical Epidemiology, Beijing, China. ${ }^{5}$ Department of Cardiology, Kailuan Hospital, North China University of Science and Technology, 57 Xinhua East Rd, Tangshan 063000, China. ${ }^{6}$ Graduate School, North China University of Science and Technology, Tangshan, China. ${ }^{7}$ School of Public Health, North China University of Science and Technology, Tangshan, China.

Received: 21 October 2020 Accepted: 30 December 2020 Published online: 12 January 2021

\section{References}

1. Roth GA, Johnson C, Abajobir A, Abd-Allah F, Abera SF, Abyu G, Ahmed M, Aksut B, Alam T, Alam K, et al. Global, regional, and national burden of cardiovascular diseases for 10 causes, 1990 to 2015. J Am Coll Cardiol. 2017;70(1):1-25.

2. Vos T, Abajobir A, Cristiana A, et al. Global, regional, and national incidence, prevalence, and years lived with disability for 328 diseases and injuries for 195 countries, 1990-2016: a systematic analysis for the Global Burden of Disease Study 2016. Lancet. 2017;390(10100):1211-59.

3. Wu S, Liu W, Ma Q, Yu W, Guo Y, Zhao Y, Shi D, Liu Y, Zhou Z, Wang J, et al. Association between insulin resistance and coronary plaque vulnerability in patients with acute coronary syndromes: insights from optical coherence tomography. Angiology. 2019;70(6):539-46.

4. Ormazabal V, Nair S, Elfeky O, Aguayo C, Salomon C, Zuñiga F. Association between insulin resistance and the development of cardiovascular disease. Cardiovasc Diabetol. 2018;17(1):122.

5. Bersch-Ferreira Â, Sampaio G, Gehringer M, Torres E, Ross-Fernandes M, da Silva J, Torreglosa C, Kovacs C, Alves R, Magnoni C, et al. Association between plasma fatty acids and inflammatory markers in patients with and without insulin resistance and in secondary prevention of cardiovascular disease, a cross-sectional study. Nutr J. 2018;17(1):26.

6. Laakso M. Is insulin resistance a feature of or a primary risk factor for cardiovascular disease? Curr Diabetes Rep. 2015;15(12):105.

7. Xun $P, W u Y, H e Q, H e K$. Fasting insulin concentrations and incidence of hypertension, stroke, and coronary heart disease: a meta-analysis of prospective cohort studies. Am J Clinic Nutr. 2013;98(6):1543-54.

8. Dahan M, Reaven G. Relationship among obesity, insulin resistance, and hyperinsulinemia in the polycystic ovary syndrome. Endocrine. 2019;64(3):685-9.

9. Khan S, Jena G. Sodium butyrate reduces insulin-resistance, fat accumulation and dyslipidemia in type-2 diabetic rat: a comparative study with metformin. Chemico-Biolog Inter. 2016;254:124-34.

10. Lee SB, Ahn CW, Lee BK, Kang S, Nam JS, You JH, Kim MJ, Kim MK, Park JS. Association between triglyceride glucose index and arterial stiffness in Korean adults. Cardiovasc Diabetol. 2018;17(1):41.

11. Irace C, Carallo C, Scavelli FB, De Franceschi MS, Esposito T, Tripolino C, Gnasso A. Markers of insulin resistance and carotid atherosclerosis. A comparison of the homeostasis model assessment and triglyceride glucose index. Int J Clin Pract. 2013;67(7):665-72.

12. Park K, Ahn CW, Lee SB, Kang S, Nam JS, Lee BK, Kim JH, Park JS. Elevated TyG index predicts progression of coronary artery calcification. Diabetes Care. 2019;42(8):1569-73.

13. Lee EY, Yang HK, Lee J, Kang B, Yang Y, Lee SH, Ko SH, Ahn YB, Cha BY, Yoon KH, et al. Triglyceride glucose index, a marker of insulin resistance, is associated with coronary artery stenosis in asymptomatic subjects with type 2 diabetes. Lipids Health Dis. 2016;15(1):155. 
14. da Silva A, Caldas APS, Hermsdorff HHM, Bersch-Ferreira ÂC, Torreglosa CR, Weber B, Bressan J. Triglyceride-glucose index is associated with symptomatic coronary artery disease in patients in secondary care. Cardiovasc Diabetol. 2019;18(1):89.

15. Xie Y, Guo R, Li Z, Guo X, Sun G, Sun Z, Zheng J, Sun Y, Zheng L. Temporal relationship between body mass index and triglyceride-glucose index and its impact on the incident of hypertension. Nutr Metab Cardiovasc Dis. 2019;29(11):1220-9.

16. Aslan Çin NN, Yardımcı H, Koç N, Uçaktürk SA, Akçil Ok M. Triglycerides/high-density lipoprotein cholesterol is a predictor similar to the triglyceride-glucose index for the diagnosis of metabolic syndrome using International Diabetes Federation criteria of insulin resistance in obese adolescents: a cross-sectional study. J Pediatr Endocrinol Metab. 2020;33(6):777-84.

17. Sánchez-Îñigo L, Navarro-González D, Fernández-Montero A, PastranaDelgado J, Martínez J. The TyG index may predict the development of cardiovascular events. Eur J Cli Invest. 2016;46(2):189-97.

18. Li S, Guo B, Chen H, Shi Z, Li Y, Tian Q, Shi S. The role of the triglyceride (triacylglycerol) glucose index in the development of cardiovascular events: a retrospective cohort analysis. Sci Rep. 2019;9(1):7320.

19. Zhao Q, Zhang TY, Cheng YJ, Ma Y, Xu YK, Yang JQ, Zhou YJ. Impacts of triglyceride-glucose index on prognosis of patients with type 2 diabetes mellitus and non-ST-segment elevation acute coronary syndrome: results from an observational cohort study in China. Cardiovasc Diabetol. 2020;19(1):108.

20. Liu Y, Wu M, Xu J, Sha D, Xu B, Kang L. Association between Triglyceride and glycose (TyG) index and subclinical myocardial injury. Nutr Metab Cardiovasc Dis. 2020. https://doi.org/10.1016/j.numecd.2020.06.019.

21. Ma X, Dong L, Shao Q, Cheng Y, Lv S, Sun Y, Shen H, Wang Z, Zhou Y, Liu X. Triglyceride glucose index for predicting cardiovascular outcomes after percutaneous coronary intervention in patients with type 2 diabetes mellitus and acute coronary syndrome. Cardiovasc Diabetol. 2020;19(1):31.

22. Hu C, Zhang J, Liu J, Liu Y, Gao A, Zhu Y, Zhao Y. Discordance between the triglyceride glucose index and fasting plasma glucose or $\mathrm{HbA1C}$ in patients with acute coronary syndrome undergoing percutaneous coronary intervention predicts cardiovascular events: a cohort study from China. Cardiovasc Diabetol. 2020;19(1):116.

23. Anderson A, Yang W, Townsend R, Pan Q, Chertow G, Kusek J, Charleston J, He J, Kallem R, Lash J, et al. Time-updated systolic blood pressure and the progression of chronic kidney disease: a cohort study. An Inter Med. 2015;162(4):258-65.

24. Wang A, Liu J, Li C, Gao J, Li X, Chen S, Wu S, Ding H, Fan H, Hou S. Cumulative exposure to high-sensitivity C-reactive protein predicts the risk of cardiovascular disease. J Am Heart Assoc. 2017;6(10):e005610.

25. Jin C, Chen S, Vaidya A, Wu Y, Wu Z, Hu FB, Kris-Etherton P, Wu S, Gao X. Longitudinal change in fasting blood glucose and myocardial infarction risk in a population without diabetes. Diabetes Care. 2017;40(11):1565-72.

26. Wang A, Sun Y, Liu X, Su Z, Li J, Luo Y, Chen S, Wang J, Li X, Zhao Z, et al. Changes in proteinuria and the risk of myocardial infarction in people with diabetes or pre-diabetes: a prospective cohort study. Cardiovasc Diabetol. 2017;16(1):104.

27. Wu S, Huang Z, Yang X, Zhou Y, Wang A, Chen L, Zhao H, Ruan C, Wu Y, Xin $A$, et al. Prevalence of ideal cardiovascular health and its relationship with the 4-year cardiovascular events in a northern Chinese industrial city. Circ Cardiovasc Qual Outcomes. 2012;5(4):487-93.

28. Thygesen K, Alpert J, Jaffe A, Chaitman B, Bax J, Morrow D, White H. Fourth Universal Definition of Myocardial Infarction. J Am Coll Cardiol. 2018;72(18):2231-64.

29. Bastard JP, Lavoie ME, Messier V, Prud'homme D, Rabasa-Lhoret R. Evaluation of two new surrogate indices including parameters not using insulin to assess insulin sensitivity/resistance in non-diabetic postmenopausal women: a MONET group study. Diabetes Metab. 2012;38(3):258-63.

30. Sánchez-García A, Rodríguez-Gutiérrez R, Mancillas-Adame L, GonzálezNava V, Díaz González-Colmenero A, Solis RC, Álvarez-Villalobos NA, González-González JG. Diagnostic accuracy of the triglyceride and glucose index for insulin resistance: a systematic review. Int J Endocrinol. 2020;2020:4678526.

31. Simental-Mendía LE, Rodríguez-Morán M, Guerrero-Romero F. The product of fasting glucose and triglycerides as surrogate for identifying insulin resistance in apparently healthy subjects. Metab Syndr Relat Disord. 2008;6(4):299-304.

32. Olsson M, Schnecke V, Cabrera C, Skrtic S, Lind M. Contemporary risk estimates of three $\mathrm{HbA} 1 \mathrm{c}$ variables for myocardial infarction in 101,799 patients following diagnosis of type 2 diabetes. Diabetes Care. 2015;38(8):1481-6.

33. Wang A, Liu X, Su Z, Chen S, Zhang N, Wu S, Wang Y, Wang Y. Two-year changes in proteinuria and the risk of stroke in the Chinese population: a prospective cohort study. J Am Heart Assoc. 2017;6(7):e006271.

34. Arshi B, Tohidi M, Derakhshan A, Asgari S, Azizi F, Hadaegh F. Sex-specific relations between fasting insulin, insulin resistance and incident hypertension: 8.9 years follow-up in a Middle-Eastern population. J Hum Hyper. 2015;29(4):260-7.

35. Millstein R, Pyle L, Bergman B, Eckel R, Maahs D, Rewers M, Schauer I, Snell-Bergeon J. Sex-specific differences in insulin resistance in type 1 diabetes: the CACTI cohort. J Diabetes Complications. 2018;32(4):418-23.

36. Lovejoy J, Champagne C, de Jonge L, Xie H, Smith S. Increased visceral fat and decreased energy expenditure during the menopausal transition. Inter J Obes. 2008;32(6):949-58.

37. Markus MRP, Rospleszcz S, Ittermann T, Baumeister SE, Schipf S, SiewertMarkus U, Lorbeer R, Storz C, Ptushkina V, Peters A, et al. Glucose and insulin levels are associated with arterial stiffness and concentric remodeling of the heart. Cardiovasc Diabetol. 2019;18(1):145.

\section{Publisher's Note}

Springer Nature remains neutral with regard to jurisdictional claims in published maps and institutional affiliations.

\footnotetext{
Ready to submit your research? Choose BMC and benefit from:

- fast, convenient online submission

- thorough peer review by experienced researchers in your field

- rapid publication on acceptance

- support for research data, including large and complex data types

- gold Open Access which fosters wider collaboration and increased citations

- maximum visibility for your research: over 100M website views per year
}

At $\mathrm{BMC}$, research is always in progress.

Learn more biomedcentral.com/submissions 